\title{
Early Intensive Physical and Respiratory Therapy after Emergency Abdominal Surgery: Pilot Phase of the Incidence of Complications Following Emergency Abdominal Surgery Get Exercising (ICEAGE) Randomised Controlled Trial.
}

\author{
lanthe Boden ( $\square$ ianthe.boden@ths.tas.gov.au ) \\ Launceston General Hospital \\ Kate Sullivan \\ Launceston General Hospital \\ Claire Hackett \\ Princess Alexandra Hospital \\ Brooke Winzer \\ Northeast Health Wangaratta \\ Rita Hwang \\ Princess Alexandra Hospital \\ David Story \\ The University of Melbourne \\ Linda Denehy \\ The University of Melbourne
}

Research Article

Keywords: Emergency laparotomy, Abdominal surgery, Physiotherapy, Postoperative recovery, Enhanced recovery after surgery (ERAS), Pilot study

Posted Date: November 8th, 2021

DOI: https://doi.org/10.21203/rs.3.rs-1041796/v1

License: @) (1) This work is licensed under a Creative Commons Attribution 4.0 International License. Read Full License 


\section{Abstract}

Background: After emergency abdominal surgery, patients are at significantly greater risk of delayed physical recovery and developing a pulmonary complication compared to patients having the equivalent elective procedure. No randomised controlled trial has assessed the feasibility, safety, or effectiveness of intensive postoperative physiotherapy in this high-risk acute population.

Methods: The internal pilot phase of the Incidence of Complications after Emergency Abdominal surgery Get Exercising (ICEAGE) trial was a prospective, parallel-group, single-centre, active-placebo controlled, randomised trial that evaluated the feasibility, safety, and clinical trial processes of providing early intensive physiotherapy following emergency abdominal surgery, prior to rolling into a planned multicentre efficacy phase. The internal pilot phase of ICEAGE recruited fifty consecutive patients having emergency abdominal surgery at the principal participating hospital. Eligible patients were randomly assigned to standard-care physiotherapy (single session of education and coached breathing exercises plus daily assisted ambulation for 15-minutes) or intensive physiotherapy (unlimited education, twice daily coached breathing exercises for two days, and 30-minutes of daily supervised rehabilitation over the first five postoperative days).

Results: Interventions were successfully provided as per-protocol in 35\% (78/221) of planned treatment sessions. Primary barriers to protocol delivery were physiotherapist unavailability on weekends (59/221 (27\%)), awaiting patient consent (18/99 (18\%)), and patient fatigue (26/221 (12\%)). Despite these inhibitors to treatment delivery, the intervention group received twice as many breathing exercise sessions and four-fold the total duration of assisted exercise over the first five postoperative days (86 minutes (IQR 53 - 121) versus 23 minutes (IQR 12 - 29), p<0.001). One adverse event was reported from 78 supervised physical activity sessions $(1.3 \%)$ which resolved fully on cessation of activity without the need for escalation of medical care.

Conclusions: Intensive postoperative physiotherapy was safely delivered within a randomised controlled trial environment and resulted in significantly more coached breathing exercises and greater time spent physically active over the first five days after emergency abdominal surgery. The multicentre phase of ICEAGE conducted within a pragmatic and widely generalisable clinical environment will provide inaugural evidence on the effectiveness of intensive physiotherapy to prevent pulmonary complications and improve physical recovery after emergency abdominal surgery.

Trial registration: ANZCTRN 12615000318583. Prospectively registered $8^{\text {th }}$ April 2015.

\section{Background}

Globally, millions of people require time-critical emergency surgery every year to resolve potentially life-limiting small bowel obstructions, peritonitis, gastrointestinal tract perforations, invasive cancerous tumours, and traumatic abdominal injuries ${ }^{1}$. Outcomes following emergency abdominal surgery are generally poorer when compared to the equivalent elective procedure ${ }^{2}$. Representing just $11 \%$ of surgical cases in the United States, emergency surgery accounts for half of all postoperative deaths and a third of all complications ${ }^{2}$. The most common major complication after emergency abdominal surgery is a postoperative pulmonary complication (PPC), occurring in $20-50 \%$ of patient $\mathrm{s}^{3-6}$. Compared to other patient and surgical factors, having emergency surgery carries the single greatest risk for developing a PPC ${ }^{5}$. PPCs are strongly associated with increased healthcare costs ${ }^{5,7}$, longer hospital stay ${ }^{6-8}$, increased mortality ${ }^{6-8}$, and delayed recovery ${ }^{10}$.

Preoperative inspiratory muscle training ${ }^{11,12}$, exercise training ${ }^{12}$, and preoperative education and breathing training from a physiotherapist ${ }^{13}$ all result in large reductions in PPC risk for patients having elective abdominal surgery, however, implementing these therapies prior to emergency abdominal surgery may not be feasible, nor effective, due to the very short period between presenting to hospital with symptoms and the operation. A postoperative phase intervention is logically a more feasible option for this cohort.

In addition to a higher risk of PPCs, both physical recovery and quality of life are significantly worse after emergency abdominal surgery ${ }^{14}$. A third of patients are unable to walk without assistance one week after surgery ${ }^{15}$ and almost half suffer ongoing functional impairment after discharge from hopsital ${ }^{16}$. A common assumption is that increased early postoperative rehabilitation could mitigate these functional deficits ${ }^{5,14,15,17}$. Although an association between delayed mobilisation and poorer outcomes following emergency abdominal surgery is evident ${ }^{15,18}$ only one small single-centre pre-post cohort trial has attempted to assess if additional physical rehabilitation can improve outcomes ${ }^{19}$. Unfortunately, despite the high incidence of PPCs and known prolonged physical dysfunction, no randomised controlled trial has investigated any type of intervention aimed at mitigating PPCs or improving physical recovery after emergency abdominal surgery ${ }^{20}$. Large, high-quality trials assessing the efficacy and safety of interventions to improve outcomes in the high-risk, high-acuity cohort of emergency abdominal surgery are urgently needed ${ }^{21}$.

The Incidence of Complications after Emergency Abdominal surgery: Get Exercising (ICEAGE) 22 trial was designed to test the effect of postoperative physiotherapy on the incidence of PPCs and rate of physical recovery after emergency abdominal surgery. The intervention is a multimodal combination of patient education, postoperative breathing exercises, and intensive assisted early rehabilitation conducted over the first five postoperative days in the intensive care unit and/or surgical ward. Considering the paucity of prior research in this high-risk acute population it is vital to ascertain if a complex exercise-based clinical trial is feasible and safe to deliver. It is also important to assess the appropriateness of trial design and conduct prior to progressing to a definitive large-scale multicentre trial 22,24 .

The objective of the single-centre internal pilot phase of ICEAGE was to assess the feasibility, safety, and processes of conducting a randomised controlled trial of daily intensive physiotherapy in the first week after emergency abdominal surgery. Results would inform a decision to continue the trial and any adaptations to study design and procedures required prior to rolling into a multicentre phase. 


\section{Methods}

Aim

The following questions were assessed: 1) do the eligibility criteria provide a representative sample of the emergency abdominal surgery population? 2) will recruitment rate be sufficient to complete the trial within reasonable time? 3) is data collection feasible? 4) are the postoperative physiotherapy protocols feasible to deliver? 5) is there a significant difference in treatment dosage between groups? 6) are the intervention arms safe compared to the benchmark of adverse event rates during physiotherapy sessions to critically unwell patients?

\section{Design and setting}

From June 2015 to October 2016, we conducted a prospective single-centre double-blinded (patient and assessor) parallel-group randomised controlled trial at a tertiary government-funded regional hospital in Australia. Trial conduct, processes, procedures, and treatment protocols were piloted for feasibility and safety to inform modifications required prior to rolling into the intended full-scale multicentre phase of ICEAGE. The pilot phase of ICEAGE was granted local ethical approval (Human Research Ethics Committee (Tasmania) Network, Tasmania, Australia (H0013666) and was prospectively registered on the $8^{\text {th }}$ of $A p r i l$ 2015 in the Australian and New Zealand Clinical Trial Registry (ANZCTRN 12615000318583) ${ }^{25}$. This manuscript is reported in accordance with the CONSORT guidelines for randomised pilot and feasibility trials ${ }^{26}$.

\section{Participants}

Eligible participants were adults ( $\geq 18$ years) admitted for emergency abdominal surgery requiring an open incision above, or extending above, the umbilicus. Patients were ineligible if they did not provide consent to participate, were preoperatively unable to walk for one minute without a seated rest, unable to understand English, received preoperative education and breathing exercise training from a physiotherapist ${ }^{13}$, had undergone elective abdominal surgery in the previous seven days, assessed by senior medical staff as approaching imminent death within 48 hours of surgery, or had medical orders not to participate in early active rehabilitation. Patients were also excluded if the research team were unable to approach the eligible patient within 48 hours of surgery.

New admissions to surgical wards and the intensive care unit were screened for eligible patients by the research team on weekdays. Eligible patients were provided with a single standardised physiotherapy session at the earliest possible occasion within the first two days after surgery. Following this session, eligible patients were approached at the earliest convenient time by the chief investigator (IB) or principal site investigator (KS). Written and verbal information about the clinical trial was provided and signed consent gained if the patient was willing to participate. If patient was incapable of consenting (i.e sedated, delirium, severe pain, cognitively impaired) the next of kin was contacted.

Following consent being gained, the treating physiotherapist opened a sequentially numbered concealed envelope pre-prepared by an independent administration assistant. Allocation order was by computer generated blocked random number table (1:1). The participant's name was written on the envelope to record that recruitment occurred in order of eligibility. Participants, hospital doctors, nurses, and assessors were masked to group allocation to group allocation. Participants were withdrawn if they withdrew consent.

\section{Treatment}

Prior to recruitment all participants received an initial standardised physiotherapy treatment session on the first available occasion within the first two postoperative days (Figure 1). This session consisted of education on PPC prevention with early ambulation and breathing exercises, assisted mobilisation out of bed, and a single session of coached deep breathing and coughing exercises. Participants were encouraged to continue performing their own breathing exercises every hour during the day until they were frequently ambulant. An information booklet was provided to reinforce the information and coaching provided. Education content, information booklet, and breathing exercises were based on those previously shown to be highly valued by patients ${ }^{27}$ and effective in halving PPC incidence after elective abdominal surgery ${ }^{13}$. Following recruitment into the trial, participants were assigned to receive ongoing standard-care physiotherapy (control) or intensive physiotherapy (intervention).

Standard-care physiotherapy (Control): Following the standardised initial physiotherapy session, control group participants were provided with once-daily assisted ambulation with a physiotherapist or with a therapy assistant working under the direct supervision of a physiotherapist. Assisted ambulation was delivered using a protocol utilised previously in elective abdominal surgery ${ }^{13,27-29}$ (Figure 1). The aim was to assist the participant to walk away from the bedside for at least three minutes to a target of 10 minutes, but no longer than 15 minutes. The total assisted ambulation per-protocol time over the first five days was 50 minutes. Assisted ambulation sessions were ceased once a threshold score determining readiness to discharge from physiotherapy was met ${ }^{30}$.

Intensive physiotherapy (Intervention): In addition to standard-care physiotherapy, intervention group participants received additional coached breathing exercises, functional rehabilitation exercises, and education (Figure 1). Participants were provided with twice-daily coached deep breathing and coughing sessions for the first two days and once-daily assisted ambulation and targeted functional and strengthening exercises. Exercise prescription was according to a descending scale dependent on patient acuity, conscious status, and functional capacity. Exercises were individualised to the participant's functional status and progressed daily by the physiotherapist. Total daily exercise therapy duration target was at least 30 minutes. The target total assisted rehabilitation per-protocol time over the first five days was 150 minutes. The ICEAGE rehabilitation protocol was modelled on previous critical care rehabilitation trials ${ }^{31,32}$. Specific exercises, repetitions, intensity, and sets were chosen pragmatically by the treating physiotherapist using their clinical judgement. Additional education regarding preventing PPCs, improving recovery, and prompts to continue with breathing exercises and physical activity were provided daily at the discretion of the treating physiotherapist. Intensive physiotherapy was provided each day for a minimum of the first five postoperative hospital days and then continued until a threshold score was $\mathrm{met}^{30}$, or day of hospital discharge, whichever came first. 


\section{Outcomes}

\section{Feasibility of recruitment}

Recruitment feasibility was measured with the following metrics; number of patients having emergency abdominal surgery, number fulfilling eligibility criteria, number of occasions and reasons for when eligibility criteria were queried by the investigating team as possibly considered unsuitable/unrepresentative and recommended for modification, number and reasons for not being able to approach patients to gain consent to enrol in study, number of patients approached within the first two postoperative days, number who consented, number who withdrew.

A convenience sample of 20 consecutive participants provided feedback regarding their experience of the recruitment process. These participants were interviewed by the chief investigator (IB) within seven days of surgery, face-to-face or by telephone, using a semi-structured interview. Interviews were collated and interpreted thematically and by proportional responses.

\section{Feasibility of data collection}

Number lost to follow-up at the 14-day primary trial outcome of PPC and 90-day secondary trial outcome of health-related quality of life.

\section{Feasibility of treatment protocols}

Feasibility of delivering the protocol was assessed by recording reasons for not being able to provide treatment protocols within the first five postoperative days and number of protocol violations.

\section{Separation between groups}

This was assessed using time from surgery to first successful assisted ambulation event $>3$ mins, total duration of exercise sessions provided over the first five postoperative days, exercise time provided as a proportion of total protocolised time (control group 50mins; intervention group 150mins), and total number of coached breathing exercise sessions provided within the first three postoperative days. Time to receive the initial physiotherapy assisted ambulation session was intended to be similar whilst a separation between groups was intended for number of coached respiratory sessions and duration of assisted physical activity over the first five postoperative days.

\section{Safety of treatment protocols}

An adverse event was defined as a physiological event that occurred in direct response to delivering the treatment protocol. These included, a) symptomatic blood pressure or heart rate change $20 \%$ from resting heart rate, new cardiac arrhythmia, and drop in oxygen saturation > $10 \%$ from baseline which fail to recover within two minutes of ceasing activity, b) requirement to increase sedation or inotropic support, c) line detachment, d) fall, or e) or severe nausea. The proportion of adverse events caused directly by the ICEAGE treatment protocols was compared to the adverse event rate of $2 \%$ in surgical critical care patients provided with assisted early mobilisation ${ }^{32}$.

No a priori hypotheses of trial conduct success or failure were set. The decision to progress with the trial was made pragmatically based on the analysed pilot data and anticipated prospective recruitment rates given appropriate modifications, if necessary, to the study protocol.

For the pilot phase of ICEAGE, between-group estimates of treatment effect to the trial's primary outcome, PPC, and key secondary outcomes were not undertaken unless there was a decision to cease the trial for futility. This was to deliberately avoid a possible detection bias that could influence ongoing trial conduct given a decision to proceed with the trial.

\section{Sample size}

The primary aim of this pilot phase was to investigate trial feasibility, safety, and trial processes rather that to estimate a treatment effect. The sample size for the ICEAGE pilot phase was pragmatically set at $100^{25,33}$. This was the expected throughput of eligible patients at the participating centre within a 12 -month period. After 12-months this size sample had not been attained. The pilot phase continued for a total of 15 months until a revised target sample of 50 was achieved.

\section{Statistical methods}

Data was analysed with intention-to-treat principles. Recruitment data was reported in numbers and proportions. Imbalances between-groups in baseline measures was assessed to determine covariates for inclusion in comparative testing for group treatment separation outcomes. Continuous measures were compared with independent samples t-tests or Mann Whitney $U$ test depending on normality (Shapiro-Wilk test). Fishers exact test was used for proportions. With equivalence established, outcomes were assessed using simple main effects comparisons between-groups with the same tests. Between-group differences in the duration of supervised physical activity each day over the first five postoperative days was analysed using two-way mixed ANOVA with nonparametric data transformed with square root functions, Bonferroni adjustment for $95 \%$ confidence intervals, and p-values estimated with Greenhouse-Geisser adjustment for non-sphericity. Simple main effects for differences between-groups on each postoperative day separately were analysed with one-way ANOVAs. Values are reported as means with standard deviations (SD) for normally distributed variables, medians with interquartile ranges for non-parametric continuous variables, and frequencies and percentages for categorical values. P values are two-sided with $<0.05$ considered statistically significant.

\section{Results}


From $24^{\text {th }}$ June 2015 to $25^{\text {th }}$ October 2016,227 patients had emergency abdominal surgery at the participating hospital. One hundred patients (44\%) had at least one exclusion criteria and were therefore not approached by investigators (Figure 2). The two most common reasons for exclusion were: received preoperative education by a physiotherapist $n=26(15 \%)$ and laparoscopic or lower abdominal surgery $n=26$ (15\%) (Figure 2). Of 127 eligible patients, one in three (45/127) could not be enrolled within 48 hours of surgery and were also excluded. This was predominantly due to unavailability of research staff over weekends or patients being in a vulnerable state (mechanically ventilated, delirious, severe nausea or pain) and researchers being unable to contact their next of kin.

Of eligible patients approached, the majority consented to participate (61\% (50/82)) with one person (2\%) withdrawing from the trial.

Twenty consecutive participants were interviewed and all (100\%) reported feeling significantly unwell in the first two to three days after surgery and would have preferred not to have been approached by researchers regarding participation in any type of clinical trial during this early postoperative period. Conversely, consensus opinion was that the ICEAGE trial had a high degree of value to them, were glad they enrolled, and wanted to continue to participate in it. Given a choice between the current consenting process of written informed consent prior to randomisation or deferred consent, all patients (100\%) preferred being automatically enrolled in the study with autonomy to consent or withdraw when approached by investigators later. The consensus recommended time to be approached regarding participating in a clinical trial was after the third postoperative day or when they were no longer nauseated or in pain.

Four of the a priori eligibility criteria were queried for modification or removal by the investigating team:

i. The exclusion criteria neglected to exclude patients with an existing PPC. Recruiting a patient who already meets the trial's primary outcome is not sensible therefore the eligibility criteria were amended within the first week of the trial to include this criterion. $7 \%$ (16/227) patients had a PPC on screening for eligibility and were therefore excluded (Figure 2).

ii. Publication of the LIPPSMAck-POP trial results ${ }^{13}$ made it undesirable to the local physiotherapy team to withhold preoperative physiotherapy education if it was possible to deliver this whilst a patient was awaiting emergency abdominal surgery. This criterion was recommended to be withdrawn.

iii. Excluding patients who had lower abdominal or minimally invasive incisions was also recommended to be retracted. During the period from protocol inception to trial initiation, advances in surgical techniques had led to increased use of minimally invasive incisions for procedures that would have previously been performed via larger incisions. Including all incision types within ICEAGE would ensure the trial was more representative of the wider emergency abdominal surgery population.

iv. From the feedback received from participants, it was proposed that the consent process be changed from informed consent prior to randomisation to a deferred consent process.

The number of patients excluded for items (ii) - (iv) totalled $43 \%$ of all patients (97/227) having emergency abdominal surgery at the primary participating centre. In combination with the patients excluded for sensible reasons and non-consenters, this provided a recruiting rate of just $22 \%$ of the total population. It was calculated that it would not be possible to recruit within a suitable timeframe the sample of 288 required for ICEAGE to be powered for superiority unless modifications to the eligibility criteria and consent process were made.

Feasibility of data collection

The trial's primary outcome, PPC, was obtained in $100 \%$ of participants by the $14^{\text {th }}$ postoperative day. There was a $50 \%$ loss to follow-up for the secondary outcome of quality of life at 90-day either through the patient being uncontactable or deceased (Figure 2).

\section{Feasibility of treatment protocols}

Participants in the two groups were equivalent at baseline in factors that may have influenced delivery of the treatment protocols, such as age, comorbidities, preoperative mobility, surgical procedure, admission to the intensive care unit (ICU), or need for ongoing postoperative mechanical ventilation (Table 1). Trial interventions were provided exactly as prescribed in $35 \%$ of all protocolised treatment events (Table 2). The most common barriers to providing the protocol were lack of physiotherapy staff available on weekends or public holidays (27\%), awaiting patient consent (18\%), patient fatigue (12\%), pain (5\%), refusal to participate in a treatment session (4\%), and hypotension (3\%). Eight (32\%) participants in the standard-care group were provided with more physiotherapy than the specified protocol with additional assisted ambulation sessions beyond the day the designated threshold score indicated that these sessions should cease (supplemental file 1). One was also provided with assisted ambulation of 20 minutes per session, longer than the protocolised maximum duration of 15 minutes. Two (8\%) participants in the intervention group were enrolled with an existing PPC in the first week of the trial prior to amendment of the eligibility criteria.

Table 1. Baseline demographic and clinical characteristics of the study population 


\begin{tabular}{|c|c|c|}
\hline & $\begin{array}{l}\text { Standard care physiotherapy } \\
(\mathrm{n}=25)\end{array}$ & $\begin{array}{l}\text { Intensive physiotherapy } \\
(\mathrm{n}=24)\end{array}$ \\
\hline Age (yr), median (IQR) & $64(57-74)$ & $70(55-76)$ \\
\hline Male, gender, n (\%) & $11(44)$ & $15(63)$ \\
\hline BMI (wt (kg)/ht $\left.(\mathrm{cm})^{2}\right)$, mean +/- SD & $25(4)$ & $27(6)$ \\
\hline \multicolumn{3}{|l|}{ ASA physical health status, n (\%) } \\
\hline $1-2$ & $4(16)$ & $7(29)$ \\
\hline $3-4$ & $14(56)$ & $14(57)$ \\
\hline No ASA recorded & $7(28)$ & $3(13)$ \\
\hline \multicolumn{3}{|l|}{ Co-morbidities, n (\%) } \\
\hline Respiratory & $11(44)$ & $10(42)$ \\
\hline Cardiac disease & $10(40)$ & $8(33)$ \\
\hline Cancer & $7(28)$ & $5(21)$ \\
\hline Arthritis & $5(20)$ & $7(29)$ \\
\hline Mental health disease & $4(16)$ & $5(21)$ \\
\hline Stroke/TIA/ neurological disease & $5(20)$ & $2(8.4)$ \\
\hline Diabetes mellitus & $1(4.0)$ & $3(13)$ \\
\hline \multicolumn{3}{|l|}{ Smoking status, n (\%) } \\
\hline Never & $10(40)$ & $10(42)$ \\
\hline Ex-smoker & $8(32)$ & $3(13)$ \\
\hline Current smoker & $7(28)$ & $11(46)$ \\
\hline Average pack years & $41(23)$ & $22(22)$ \\
\hline Preoperative mobility limitation, n (\%) & $6(24)$ & $9(38)$ \\
\hline Preoperative HrQOL (WHODAS), median (IQR) & $16(14-29)$ & $14(12-25)$ \\
\hline \multicolumn{3}{|l|}{ Primary reason for surgery } \\
\hline Organ dysfunction & $22(88)$ & $22(92)$ \\
\hline Trauma & $3(12)$ & $2(8)$ \\
\hline \multicolumn{3}{|l|}{ Surgical procedure, $\mathrm{n}(\%)$} \\
\hline Bowel resection & $5(32)$ & $10(42)$ \\
\hline Laparotomy - adhesiolysis & $8(32)$ & $4(17)$ \\
\hline Laparotomy - explorative & $2(8.0)$ & $3(13)$ \\
\hline Vascular & $7(28)$ & $1(4.2)$ \\
\hline Cholecystectomy & $0(0)$ & $3(13)$ \\
\hline Gastrectomy & $0(0)$ & $2(8.3)$ \\
\hline Pancreatectomy & $0(0)$ & $1(4.2)$ \\
\hline \multicolumn{3}{|l|}{ Incision, n (\%) } \\
\hline Midline laparotomy & $24(96)$ & $21(88)$ \\
\hline Transverse & $1(4.0)$ & $3(12)$ \\
\hline Operation duration (mins), mean +/- SD & $130(54)$ & $151(68)$ \\
\hline ICU admission, n (\%) & $9(36)$ & $9(38)$ \\
\hline Mechanically ventilated Day $1, n(\%)$ & $5(20)$ & $4(17)$ \\
\hline \multicolumn{3}{|l|}{ Data are mean (SD), median (IQR), or $\mathrm{n}(\%)$. } \\
\hline \multicolumn{3}{|c|}{$\begin{array}{l}\text { BMI=Body Mass Index, ASA=American Society Anaesthesiologists, TIA=transient ischaemic attack, HrQOL=health related quality of life, WHODAS=World } \\
\text { Health Organisation disability assessment schedule, ICU=intensive care unit }\end{array}$} \\
\hline
\end{tabular}


Table 2: Treatment protocol delivery and barriers over first five postoperative days

\begin{tabular}{|c|c|c|c|c|c|c|c|c|c|c|c|}
\hline \multirow{2}{*}{$\begin{array}{l}\text { Postoperative } \\
\text { day }\end{array}$} & \multicolumn{2}{|l|}{ Day 1} & \multicolumn{2}{|l|}{ Day 2} & \multicolumn{2}{|l|}{ Day 3} & \multicolumn{2}{|l|}{ Day 4} & \multicolumn{2}{|l|}{ Day 5} & \\
\hline & $\begin{array}{l}\text { Standard } \\
\text { care }\end{array}$ & $\begin{array}{l}\text { Intensive } \\
\text { Physio }\end{array}$ & $\begin{array}{l}\text { Standard } \\
\text { care }\end{array}$ & $\begin{array}{l}\text { Intensive } \\
\text { Physio }\end{array}$ & $\begin{array}{l}\text { Standard } \\
\text { care }\end{array}$ & $\begin{array}{l}\text { Intensive } \\
\text { Physio }\end{array}$ & $\begin{array}{l}\text { Standard } \\
\text { care }\end{array}$ & $\begin{array}{l}\text { Intensive } \\
\text { Physio }\end{array}$ & $\begin{array}{l}\text { Standard } \\
\text { care }\end{array}$ & $\begin{array}{l}\text { Intensive } \\
\text { Physio }\end{array}$ & \\
\hline & $n=25$ & $n=24$ & $n=25$ & $n=24$ & $n=24$ & $n=22$ & $n=23$ & $n=20$ & $\mathrm{n}=18$ & $n=16$ & $\begin{array}{l}\text { Total o } \\
\text { days }\end{array}$ \\
\hline $\begin{array}{l}\text { Daily number } \\
\text { discharged from } \\
\text { hospital, } n\end{array}$ & 0 & 0 & 0 & 0 & 1 & 2 & 1 & 2 & 5 & 4 & $15 / 49$ ( \\
\hline $\begin{array}{l}\text { Daily number of } \\
\text { prescribed } \\
\text { protocol sessions, } \\
n\end{array}$ & 25 & 24 & 25 & 24 & 24 & 22 & 23 & 20 & 18 & 16 & 221 \\
\hline $\begin{array}{l}\text { Daily number of } \\
\text { sessions } \\
\text { delivered as per } \\
\text { protocol, } \mathrm{n}(\%)\end{array}$ & $7(28 \%)$ & $3(13 \%)$ & $8(33 \%)$ & $9(38 \%)$ & $4(17 \%)$ & $11(50 \%)$ & $7(30 \%)$ & $12(60 \%)$ & 7 (39\%) & $10(63 \%)$ & $\begin{array}{l}78 / 221 \\
(35 \%)\end{array}$ \\
\hline \multicolumn{12}{|c|}{$\begin{array}{l}\text { Reasons that sessions were not delivered as per } \\
\text { protocol, } \mathrm{n}(\%)\end{array}$} \\
\hline $\begin{array}{l}\text { Physiotherapist } \\
\text { unavailable }\end{array}$ & $2(8 \%)$ & $6(25 \%)$ & $5(20 \%)$ & $5(21 \%)$ & $13(54 \%)$ & $6(27 \%)$ & $9(39 \%)$ & $3(15 \%)$ & $7(39 \%)$ & $3(20 \%)$ & $\begin{array}{l}59 / 221 \\
(27 \%)\end{array}$ \\
\hline Awaiting consent & $0(0 \%)$ & $12(50 \%)$ & $0(0 \%)$ & $6(25 \%)$ & - & - & - & - & - & - & $18 / 99$ ( \\
\hline Patient fatigue & $4(16 \%)$ & 0 & $4(16 \%)$ & 0 & $4(17 \%)$ & $1(5 \%)$ & $6(26 \%)$ & $3(15 \%)$ & $2(11 \%)$ & $2(13 \%)$ & $\begin{array}{l}26 / 221 \\
(12 \%)\end{array}$ \\
\hline Pain & $3(12 \%)$ & 0 & $3(12 \%)$ & $1(4 \%)$ & $1(4 \%)$ & $1(5 \%)$ & 0 & $1(5 \%)$ & 0 & 0 & $10 / 221$ \\
\hline $\begin{array}{l}\text { Declined } \\
\text { treatment }\end{array}$ & 0 & 0 & $1(4 \%)$ & $2(8 \%)$ & 0 & $3(14 \%)$ & 0 & $1(5 \%)$ & $1(6 \%)$ & 0 & $8 / 221($ \\
\hline Hypotension & $4(16 \%)$ & 0 & $2(8 \%)$ & 0 & 0 & 0 & 0 & 0 & 0 & 0 & $6 / 221($ \\
\hline $\begin{array}{l}\text { Patient } \\
\text { unavailable }\end{array}$ & $1(4 \%)$ & $1(4 \%)$ & 0 & 0 & 0 & 0 & $1(4 \%)$ & 0 & 0 & $1(7 \%)$ & $4 / 221($ \\
\hline Nausea/vomiting & $3(12 \%)$ & 0 & $1(4 \%)$ & 0 & $1(4 \%)$ & 0 & 0 & 0 & 0 & 0 & $5 / 221($ \\
\hline $\begin{array}{l}\text { Medically } \\
\text { unstable }\end{array}$ & 0 & $1(4 \%)$ & 0 & 0 & $1(4 \%)$ & 0 & 0 & 0 & 0 & 0 & $2 / 221($ \\
\hline Medical orders & 0 & $1(4 \%)$ & 0 & $1(4 \%)$ & 0 & 0 & 0 & 0 & 0 & 0 & $2 / 221($ \\
\hline $\begin{array}{l}\text { Mechanically } \\
\text { ventilated }\end{array}$ & $1(4 \%)$ & 0 & $1(4 \%)$ & 0 & 0 & 0 & 0 & 0 & 0 & 0 & $2 / 221($ \\
\hline Delirium & 0 & 0 & 0 & 0 & 0 & 0 & 0 & 0 & $1(6 \%)$ & 0 & $1 / 221($ \\
\hline Unknown & 0 & 0 & $1(4 \%)$ & 0 & 0 & 0 & 0 & 0 & 0 & 0 & $1 / 221($ \\
\hline
\end{tabular}

\section{Separation between groups for treatment protocols}

The intervention group received four times the amount of supervised physical activity and twice as many coached breathing exercises over the first five postoperative days (Table 3).

Table 3. Difference between groups in delivery of treatment protocols and adverse event rates. 


\begin{tabular}{|llll|}
\hline & $\begin{array}{l}\text { Standard care } \\
(\mathrm{n}=25)\end{array}$ & $\begin{array}{l}\text { Intensive } \\
\text { physiotherapy (n=24) }\end{array}$ & $\begin{array}{l}\mathrm{P} \\
\text { value }\end{array}$ \\
\hline Time from end of surgery to first assisted ambulation session (hrs), median (IQR) & $23(19-51)$ & $26(18-46)$ & 0.48 \\
\hline Ambulated >3mins on first postop day, $\mathrm{n}(\%)$ & $13(52 \%)$ & $14(58 \%)$ & 0.78 \\
\hline $\begin{array}{l}\text { Total duration of assisted exercise over the first five postoperative days (mins), median } \\
\text { (IQR) }\end{array}$ & $23(12-29)$ & $86(53-121)$ & $<0.001$ \\
\hline $\begin{array}{l}\text { Total exercise time over the first five days as a proportion of the target protocol, median } \\
\text { (IQR) }\end{array}$ & $\begin{array}{l}48 \%(27 \% \text { to } \\
86 \%)\end{array}$ & $62 \%(43 \%$ to $88 \%)$ & 0.29 \\
\hline Number of prophylactic coached breathing exercise sessions, median (IQR) & $1(1-1)$ & $2(0.3-3)$ & 0.02 \\
\hline Adverse events & $0(0 \%)$ & $1(4 \%)$ & 0.49 \\
\hline
\end{tabular}

As intended, both groups received their initial assisted ambulation session at a similar time point after surgery at a median time of 25 hours (IQR 19 - 48) with half of all participants able to ambulate away from the bedside for more than three minutes on the first postoperative day (Table 3). There was a significant separation between groups in the total amount of supervised physical activity provided over the first five postoperative days (standard-care physiotherapy, 23 mins (IQR 12 - 29), versus intensive physiotherapy, 86 mins (IQR 53 - 121), p<0.001; Table 3). The difference between groups was evident from the second day (Figure 3). There was no difference between groups in the total amount of exercise time delivered as a proportion of the target protocol (Table 3).

\section{Safety of treatment protocols}

From 78 supervised physical activity sessions across both groups, one participant (1.3\%) in the intervention group experienced excessive tachycardia and cardiac arrythmias in response to physical activity which fully resolved within 10 minutes of activity cessation. No escalation of medical or pharmaceutical management was required. This is no worse than the adverse event rate within critical care patients provided with early goal-directed mobilisation ( $2 \%$ )

\section{Discussion}

This pilot phase of ICEAGE was the first randomised controlled trial conducted in intensive care and surgical wards to evaluate the feasibility and safety of providing early intensive rehabilitation and breathing exercises in the high-risk, acutely unwell population following emergency abdominal surgery. It found that assisted early ambulation and rehabilitation exercises could be safely delivered within 24 hours of surgery and continued over the first five postoperative days. Despite just one in three treatment sessions being delivered as protocolised, a significant separation between groups was realised with the intervention group receiving four-fold the amount of assisted physical activity and double the coached respiratory therapy over the first five postoperative days. Given the success in obtaining a large treatment difference between groups and assured safety of the interventions it was decided to continue with ICEAGE and roll into the large-scale multicentre phase, provided modifications were made to trial conduct, consenting processes, and eligibility criteria.

To ensure that the final cohort was more representative of the broader emergency surgery population, several modifications to eligibility criteria were recommended. Advances in perioperative practice and release of new evidence in the time from trial inception to trial initiation had led to $23 \%$ of emergency abdominal surgery patients being prepared preoperatively by a physiotherapist ${ }^{13}$ or having minimally invasive incisions. Continuing to exclude these patients would lead to a population that was less representative. Given the large risk reduction in PPC associated with preoperative physiotherapy ${ }^{13}$ and desire by patients for preoperative information ${ }^{27,34}$ there were additional ethical considerations of withholding this intervention from patients awaiting emergency abdominal surgery simply so they could be included in ICEAGE. These exclusion criteria were withdrawn following ethics committee approval.

Another significant factor that diminished trial feasibility and generalisability was the significant proportion of eligible patients who were missed for recruitment within 48 hours of surgery (35\%). In the first two days after emergency surgery patients are often in a vulnerable state and without capacity to provide informed consent with the next-of-kin also in an emotionally vulnerable situation ${ }^{34}$. Previous qualitative studies report emergency surgery patients trust the hospital to do the best for them in their situation, including research, where they trust the team would not consider them for a study that would harm them ${ }^{35}$. Similarly, our participants wanted to be involved in high-value research aimed at improving postoperative recovery with deferred consent preferrable to prospective consent. Deferred consent would allow for eligible patients to be automatically enrolled, intervention treatments started without delay, and minimise patient and next-of-kin distress or discomfort by being approached by researchers when in an emotionally and physically vulnerable state ${ }^{36}$. An application to amend the recruitment process to deferred consent was tended to the local ethics committee and approved. Amending the inclusion criteria and consenting process would almost double the number of eligible patients to be recruited into the trial, greatly increasing the feasibility of completing the trial in a reasonable time frame and ensuring results are generalisable to the greater population.

At first glance, a 35\% protocol adherence rate would appear poor. However, inspection of the reasons for protocol 'failure' finds these are concordant with those previously reported as associated with poor adherence to early postoperative rehabilitation after emergency abdominal surgery ${ }^{15,17,37-39}$, namely patientreported fatigue and lack of physiotherapy staffing on weekends ${ }^{39}$. Rather than being a failure of trial conduct this should be therefore considered as inkeeping with pragmatic clinical trials principles where a study is conducted within the clinical reality of patient symptoms and capacity, and where many hospitals do not have a routine physiotherapy service on weekends ${ }^{39}$. Despite these barriers a large separation between groups was successfully achieved in this pilot trial, a feat which has proven difficult to achieve in similar trials of early rehabilitation in critical care patients ${ }^{40,41}$. An inability to deliver large differences in physical activity dosage has been suggested as a possible reason for the failure to detect a benefit favouring increased rehabilitation in these

Page 8/13 
trials ${ }^{42}$. Due to the large separation between groups detected in the pilot phase of ICEAGE a decision was made to continue the trial without specifically increasing physiotherapy staffing on the weekends or modifying the treatment protocols. This was to ensure that future results are pragmatic and reflective of the clinical reality of usual care where patients are frequently unable to participate in rehabilitation activities due to acute symptoms ${ }^{38}$ and where there may be disparate levels of resources available on weekdays versus weekends ${ }^{39}$.

\section{Conclusion}

The internal pilot phase of the ICEAGE trial found that early intensive physiotherapy of coached breathing exercises and 30-minutes of assisted physical activity delivered daily in the first five days after emergency abdominal surgery was safe and feasible, resulting in a significant increase to total dosage of breathing exercises and physical activity duration in the first week after surgery. Given the large separation in treatment dosage between groups, approved changes to eligibility criteria to improve generalisability, and allowance to enact a deferred consent process, ICEAGE was rolled into a definitive multi-centre phase in order to provide world-first evidence on the effectiveness of an intervention to prevent PPCs and improve physical recovery after emergency abdominal surgery.

\section{Abbreviations}

ICEAGE = Incidence of Complications Emergency Abdominal surgery Get Exercising

$\mathrm{IQR}=$ interquartile range

ANZCTRN = Australian and New Zealand Clinical Trial Registration Number

$\mathrm{PPC}=$ postoperative pulmonary complication

Mins $=$ minutes

ANOVA = analysis of variance

$\mathrm{SD}=$ standard deviation

LIPPSMAck-POP = Lung Infection Prevention Post Surgery Major Abdominal with Pre-Operative Physiotherapy

$\mathrm{ICU}=$ intensive care unit

\section{Declarations}

\section{Ethics approval and consent to participate}

The pilot phase of ICEAGE was granted local ethical approval (Human Research Ethics Committee (Tasmania) Network, Tasmania, Australia (H0013666). All participants, or their legal medical decision, provided written informed consent prior to randomisation. Participants were free to withdrawn from the trial at any time without reason.

\section{Consent for publication}

Not applicable

\section{Availability of data and materials}

The datasets generated and analysed during the internal pilot phase of ICEAGE are not yet publicly available due awaiting completion of the multicentre phase of ICEAGE. The physiotherapy protocol delivery details of the current dataset is available from the corresponding author on reasonable request. Once the primary ICEAGE paper is published, the dataset pertaining to this internal pilot phase will be submitted to be published as an on-line supplement to the pilot phase paper.

\section{Competing interests}

The authors declare no competing interests either financial or non-financial.

\section{Funding}

This study is an investigator-initiated trial funded by a research grant from the Clifford Craig Foundation, Launceston, Australia (Project number $130, \$ 80,000$ AUD). The funding body has no influence on the study design, collection or data analysis or publication of results. The Tasmanian Health Service - North, Tasmania, Australia, sponsored the trial through the employment of the investigators and all clinical staff related to the trial. Sponsors did not contribute to trial design, data collection, or trial management. Publications relating to the trial can be submitted without permission or requiring approval.

\section{Authors' Contributions}


IB and KS conceived and designed the study and devised the protocol. IB is the chief investigator, overseeing and coordinating trial conduct. IB and KS were site investigators and were responsible for recruitment and all trial processes at the principal site. IB and RH conducted the pilot phase data analysis and interpreted the data. IB prepared the first draft of the manuscript and was responsible for the final manuscript. All authors (IB, KS, CH, RH, BW, DS, and LD) revised critically all manuscript drafts, approved the final manuscript and contributed intellectually important content. IB is the guarantor of the paper and takes responsibility for the integrity of the work, from inception to published article.

\section{Acknowledgements}

Rebecca Lane, Jenny Keating, and Elizabeth H Skinner assisted in early phase study design and protocol conceptualisation. We would like to thank the physiotherapists and allied health assistants at the Launceston General Hospital for providing the interventions and Bronte Biggins-Tosch for working diligently on daily data collection.

\section{References}

1. Stewart B, Khanduri P, McCord C, Ohene-Yeboah M, Uranues S, Vega Rivera F, Mock C. Global disease burden of conditions requiring emergency surgery. Br J Surg. 2014;101:e9-22.

2. Smith M, Hussain A, Xiao J, Scheidler W, Reddy H, Olugbade K Jr, Cummings D, Terjimanian M, Krapohl G, Waits SA, et al. The importance of improving the quality of emergency surgery for a regional quality collaborative. Ann Surg. 2013;257:596-602.

3. Tengberg LT, Cihoric M, Foss NB, Bay-Nielsen M, Gögenur I, Henriksen R, Jensen TK, Tolstrup MB, Nielsen LB. Complications after emergency laparotomy beyond the immediate postoperative period - a retrospective, observational cohort study of 1139 patients. Anaesthesia. 2017;72:309-16.

4. Serejo LG, da Silva-Júnior FP, Bastos JP, de Bruin GS, Mota RM, de Bruin PF. Risk factors for pulmonary complications after emergency abdominal surgery. Resp Med. 2007;101:808-13.

5. Bailey JG, Davis PJ, Levy AR, Molinari M, Johnson PM. The impact of adverse events on health care costs for older adults undergoing nonelective abdominal surgery. Can J Surg. 2016;59:172-9.

6. Fernandez-Bustamante A, Frendl G, Sprung J, Kor DJ, Subramaniam B, Martinez Ruiz R, Lee JW, Henderson WG, Moss A, Mehdiratta N, et al, Perioperative Research Network Investigators. Postoperative pulmonary complications, early mortality, and hospital stay following noncardiothoracic surgery: a multicenter study by the Perioperative Research Network Investigators. JAMA Surg. 2017;152:157-66.

7. Thompson DA, Makary MA, Dorman T, Pronovost PJ. Clinical and economic outcomes of hospital acquired pneumonia in intra-abdominal surgery patients. Ann Surg. 2006;243:547-52.

8. Fleisher LA, Linde-Zwirble WT. Incidence, outcome, and attributable resource use associated with pulmonary and cardiac complications after major small and large bowel procedures. Perioper Med (Lond). 2014;3:7.

9. Arenal JJ, Bengoechea-Beeby M. Mortality associated with emergency abdominal surgery in the elderly. Can J Surg. 2003;46:111-16.

10. Tahiri M, Sikder T, Maimon G, Teasdale D, Hamadani F, Sourial N, Feldman LS, Guralnick J, Fraser SA, Demyttenaere S, et al. The impact of postoperative complications on the recovery of elderly surgical patients. Surg Endosc. 2016;30:1762-70.

11. Kendall F, Oliveira J, Peleteiro B, Pinho P, Bastos PT. Inspiratory muscle training is effective to reduce postoperative pulmonary complications and length of hospital stay: a systematic review and meta-analysis. Disability and Rehabilitation. 2018;40:864-82.

12. Assouline B, Cools E, Schorer R, Kayser B, Elia N, Licker M. Preoperative exercise training to prevent postoperative pulmonary complications in adults undergoing major surgery. A systematic review and meta-analysis with trial sequential analysis. Ann Am Thorac Soc. 2021;18:678-88.

13. Boden I, Skinner EH, Browning L, Reeve J, Anderson L, Hill C, Robertson IK, Story D, Denehy L. Preoperative physiotherapy for the prevention of respiratory complications after upper abdominal surgery: pragmatic, double blinded, multicentre randomised controlled trial. BMJ. 2018;360:j5916.

14. Sikder T, Sourial N, Maimon G, Tahiri M, Teasdale D, Bergman H, Fraser SA, Demyttenaere S, Bergman S. Postoperative recovery in frail, pre-frail, and nonfrail elderly patients following abdominal surgery. World J Surg. 2019;43:415-24.

15. Jønsson LR, Ingelsrud LH, Tengberg LT, Bandholm T, Foss NB, Kristensen MT. Physical performance following acute high-risk abdominal surgery: a prospective cohort study. Can J Surg. 2017;60:12616.

16. Tolstrup MB, Thorup T, Gögenur I. Chronic pain, quality of life and functional impairment after emergency laparotomy. World J Surg. 2019;43:161-68.

17. Burcharth J, Abdulhady L, Danker J, Ekeloef S, Jørgensen T, Lauridsen H, Lunen TB, Lyngesen M, Puggaard I, Mathiesen O, Gögenur I. Implementation of a multidisciplinary perioperative protocol in major emergency abdominal surgery. Eur J Trauma Emerg Surg. 2021;47:467-477.

18. Pederson JL, Padwal RS, Warkentin LM, Holroyd-Leduc JM, Wagg A, Khadaroo RG. The impact of delayed mobilization on post-discharge outcomes after emergency abdominal surgery: A prospective cohort study in older patients. PLoS One. 2020;15:e0241554.

19. McComb A, Warkentin LM, McNeely ML, Khadaroo RG. Development of a reconditioning program for elderly abdominal surgery patients: the Elder-friendly Approaches to the Surgical Environment-BEdside reconditioning for Functional ImprovemenTs (EASE-BE FIT) pilot study. World J Emerg Surg. 2018;13:21.

20. Sullivan K, Reeve J, Boden I, Lane R. Physiotherapy following emergency abdominal surgery. In: Garbuzenko D. eds. Actual Problems of Emergency Abdominal Surgery: InTechOpen; 2016. doi: 10.5772/63969.

21. Aggarwal G, Peden CJ, Quiney NF. Improving outcomes in emergency general surgery patients: what evidence is out there? Anesth Analg. 2017;125:14035 . 
22. Boden I, Sullivan K, Hackett C, Winzer B, Lane R, McKinnon M, Robertson I. ICEAGE (Incidence of Complications following Emergency Abdominal surgery: Get Exercising): study protocol of a pragmatic, multicentre, randomised controlled trial testing physiotherapy for the prevention of complications and improved physical recovery after emergency abdominal surgery. World J Emerg Surg. 2018;13:29.

23. Fairhurst K, Blazeby JM, Potter S, Gamble C, Rowlands C, Avery KNL. Value of surgical pilot and feasibility study protocols. Br J Surg. 2019;106:968-78.

24. El-Kotob R, Giangregorio LM. Pilot and feasibility studies in exercise, physical activity, or rehabilitation research. Pilot Feasibility Stud. $2018 ; 4: 137$.

25. Australian and New Zealand Clinical Trials Registry: Sydney (NSW): NHMRC Clinical Trials Centre, University of Sydney (Australia); 2005 - Identifier ACTRN 1261500318583. In adults following emergency upper abdoinal surgery, does an enhanced rehabilitation program with a Physiotherapist reduce the postoperative pulmonary complication rate, compared to standard ward care?; 2015 Apr 08 [cited 2021 June 18]; Available from https://www.anzctr.org.au/Trial/Registration/TrialReview.aspx?id=365543\&showOriginal=true\&isReview=true.

26. Eldridge SM, Chan CL, Campbell MJ, Bond CM, Hopewell S, Thabane L, Lancaster GA; PAFS consensus group. CONSORT 2010 statement: extension to randomised pilot and feasibility trials. BMJ. 2016;355:i5239

27. Boden I, El-Ansary D, Zalucki N, Robertson IK, Browning L, Skinner EH, L Denehy.Physiotherapy education and training prior to upper abdominal surgery is memorable and has high treatment fidelity: a nested mixed-methods randomised-controlled study. Physiotherapy. 2018;104:194-202

28. Boden I, Browning L, Skinner EH, Reeve J, El-Ansary D, Robertson IK, et al. The LIPPSMAck POP (Lung Infection Prevention Post Surgery-Major Abdominalwith Pre-Operative Physiotherapy) trial: study protocol for a multi-centre randomised controlled trial. 2015;16:1-15.

29. Lockstone J, Boden I, Robertson IK, Story D, Denehy L, Parry SM. Non-Invasive Positive airway Pressure thErapy to Reduce Postoperative Lung complications following Upper abdominal Surgery (NIPPER PLUS): protocol for a single-centre, pilot, randomised controlled trial. BMJ Open. 2019;9:e023139.

30. Brooks D, Parsons J, Newton J, Dear C, Silaj E, Sinclair L, Quirt J. Discharge criteria from perioperative physical therapy. 2002;121:488-94.

31. Hodgson CL, Bailey M, Bellomo R, Berney S, Buhr H, Denehy L, Gabbe B, Harrold M, Higgins A, Iwashyna TJ, et al; Trial of Early Activity and Mobilization Study Investigators. A binational multicenter pilot feasibility randomized controlled trial of early goal-directed mobilization in the ICU. Crit Care Med. 2016;44:1145-52.

32. Schaller SJ, Anstey M, Blobner M, Edrich T, Grabitz SD, Gradwohl-Matis I, Heim M, Houle T, Kurth T, Latronico N, et al; International Early SOMS-guided Mobilization Research Initiative. Early, goal-directed mobilisation in the surgical intensive care unit: a randomised controlled trial. 2016;388:1377-88.

33. Sim J, Lewis M. The size of a pilot study for a clinical trial should be calculated in relation to considerations of precision and efficiency. $J$ Clin Epidemiol. 2012;65:301-8.

34. Park B, Barazanchi A, Rahiri JL, Xia W, Taneja A, Hill AG. Patient Experiences of the Emergency Laparotomy Pathway: A Qualitative Study. World J Surg. 2021;45:1362-1369.

35. Tutton E, Achten J, Lamb SE, Willett K, Costa ML; UK WOLLF Research Collaborators. Participation in a trial in the emergency situation: a qualitative study of patient experience in the UK WOLLF trial. 2018;19:328.

36. Costa ML, Tutton E, Achten J, Grant R, Slowther AM. Informed consent in the context of research involving acute injuries and emergencies. Bone Joint J. 2017;99-B:147-150.

37. Paduraru M, Ponchietti L, Casas IM, Svenningsen P, Zago M. Enhanced Recovery after Emergency Surgery: A Systematic Review. Bull Emerg Trauma. 2017;5:70-78.

38. Burcharth J, Falkenberg A, Oreskov JO, Ekeloef S, Gögenur I. Patients' perceptions of barriers to enhanced recovery after emergency abdominal surgery. Langenbecks Arch Surg. 2021;406:405-412.

39. Kothari AN, Zapf MA, Blackwell RH, Markossian T, Chang V, Mi Z, Gupta GN, Kuo PC. Components of hospital perioperative infrastructure can overcome the weekend effect in urgent general surgery procedures. Ann Surg. 2015;262:683-91.

40. Wright SE, Thomas K, Watson G, Baker C, Bryant A, Chadwick TJ, Shen J, Wood R, Wilkinson J, Mansfield L, et al. Intensive versus standard physical rehabilitation therapy in the critically ill (EPICC): a multicentre, parallel-group, randomised controlled trial. 2018;73:213-221.

41. Denehy L, Skinner EH, Edbrooke L, Haines K, Warrillow S, Hawthorne G, Gough K, Hoorn SV, Morris ME, Berney S. Exercise rehabilitation for patients with critical illness: a randomized controlled trial with 12 months of follow-up. Crit Care. 2013 24;17:R156.

42. Tipping CJ, Harrold M, Holland A, Romero L, Nisbet T, Hodgson CL. The effects of active mobilisation and rehabilitation in ICU on mortality and function: a systematic review. Intensive Care Med. 2017;43:171-183.

\section{Figures}


Standardised initial physiotherapy session (all participants before randomisation)

Provided by a physiotherapist as soon as possible within the first two postoperative days.

(i) Face to face education and education booklet on prevention of postoperative pulmonary complications with breathing exercises and early ambulation.

(ii) Single session of coached deep breathing and coughing exercises. 10 supervised slow flow deep breaths to total lung capacity with a 3-5 second end inspiratory hold completed with 2 end inspiratory sniffs. 2-3 supported coughs or huffs. Repeated for another set. Patients instructed to perform independently hourly. (iii) Assisted ambulation according to the following protocol:

Stage 1 (Safety stage) Sit over edge of bed/sit in chair minimum of 2 minutes.

Stage 2 (Safety stage) March on spot 0-1 minute.

Stage 3 (Ambulation) March on spot/walk away from bedside $1-3$ minutes.

Stage 4 (Ambulation) March on spot/walk away from bedside 3-6 minutes.

Stage 5 (Ambulation) Walk away from bedside $6-10$ minutes.

Stage 6 (Ambulation) Walk away from bedside $10-15$ minutes.

Stage 7 (Ambulation) Walk away from bedside $>15$ minutes.

Aim to progress through each stage in sequence, aiming to achieve a minimum of Stage 4 with a goal for Stage 6 at a rating of perceived exertion of more than $3 / 10$.

Interval training is permissible to obtain target walking time. Each interval of rest time must not exceed the preceding work time. Total session time is the accumulative work time.

\begin{tabular}{l|l} 
Standard-care physiotherapy (control) & Intensive physiotherapy (intervention)
\end{tabular}

\begin{tabular}{l|l} 
1. Ambulation as per protocol above & 1. Additional education at discretion of physiotherapist
\end{tabular}

2. Deep breathing and coughing exercises as per

protocol above; twice daily for the first two days.

3. Ambulation as per protocol above

4. Rehabilitation exercises (i) - (v) to accumulate 30

minutes of total daily physical activity time.

(i) Lower limb closed-chain weight bearing exercises in standing,

(ii) Upper limb or lower limb exercises in sitting,

(iii) Dynamic sitting balance exercises

(iv) Active or active-assisted bed exercises

(v) Passive mobilisation or electrical muscle stimulation

Provide daily until threshold 'readiness to cease physiotherapy' score is met.

Provide daily for a minimum first five postoperative days then cease thereafter once threshold 'readiness to cease physiotherapy' score is met.

Provided by a physiotherapist or an allied health assistant under the supervision of a physiotherapist.

Aim for $>10$ minutes and no more than 15 minutes

(Stage 6) at a rating of perceived exertion of more than

$3 / 10$.

Provided by a physiotherapist.

Start physical activity session at the highest level that patient can achieve based on acuity and clinical status.

Aim for assisted ambulation for $>15$ minutes (Stage 7) at

a rating of perceived exertion of more than $3 / 10$.

Aim for at total accumulated time of at least 30 minutes at the highest degree of activity possible.

If patient fatigues prior to 30 minutes, progress

sequentially down the scale (i to $v$ ) to provide physical

activity until a total of 30 mins of activity is accumulated.

\section{Figure 1}

ICEAGE pilot phase treatment protocols 


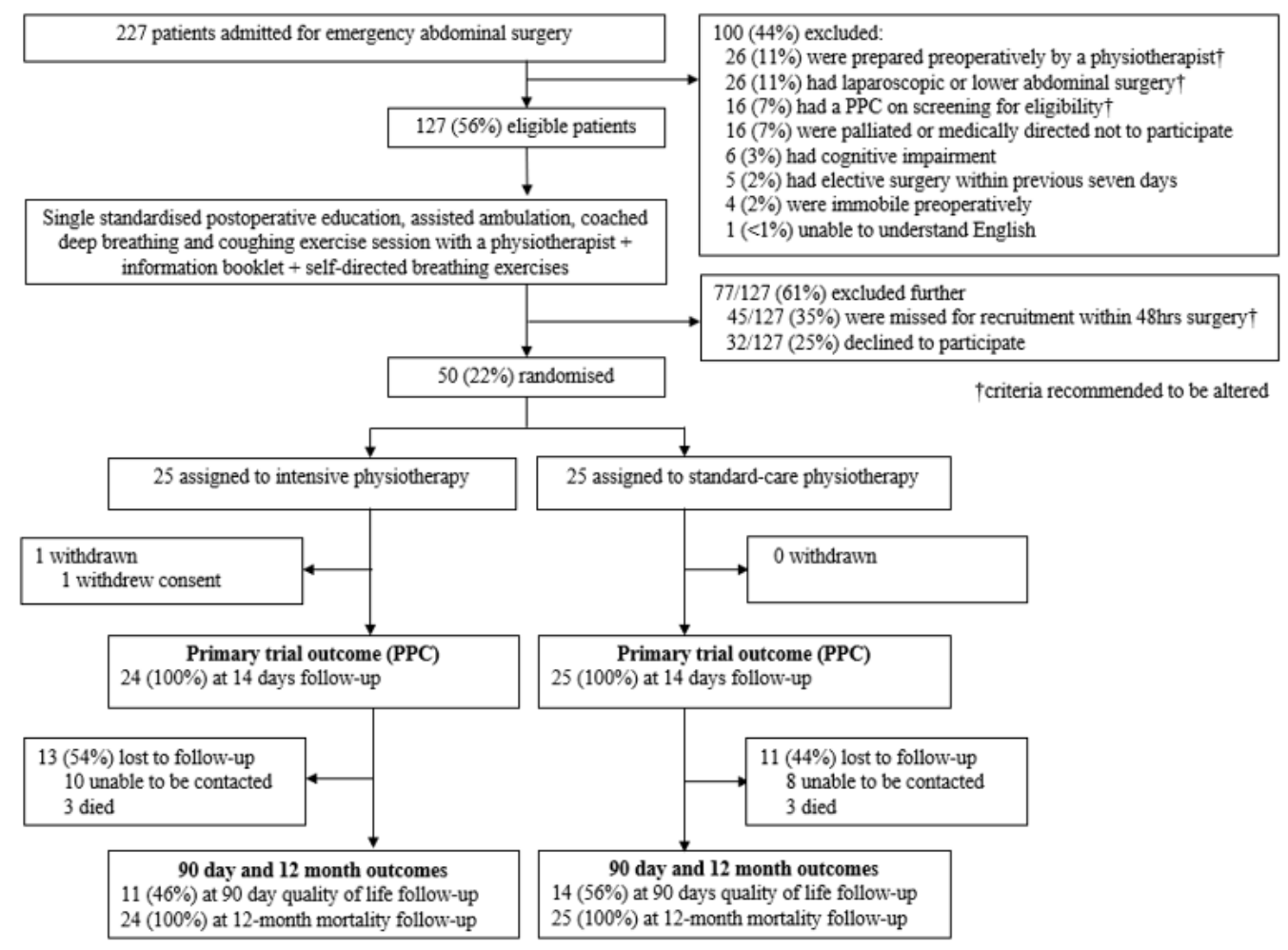

Figure 2

CONSORT flow diagram of pilot phase of ICEAGE Legend: PPC = postoperative pulmonary complication; tcriteria recommended to be altered

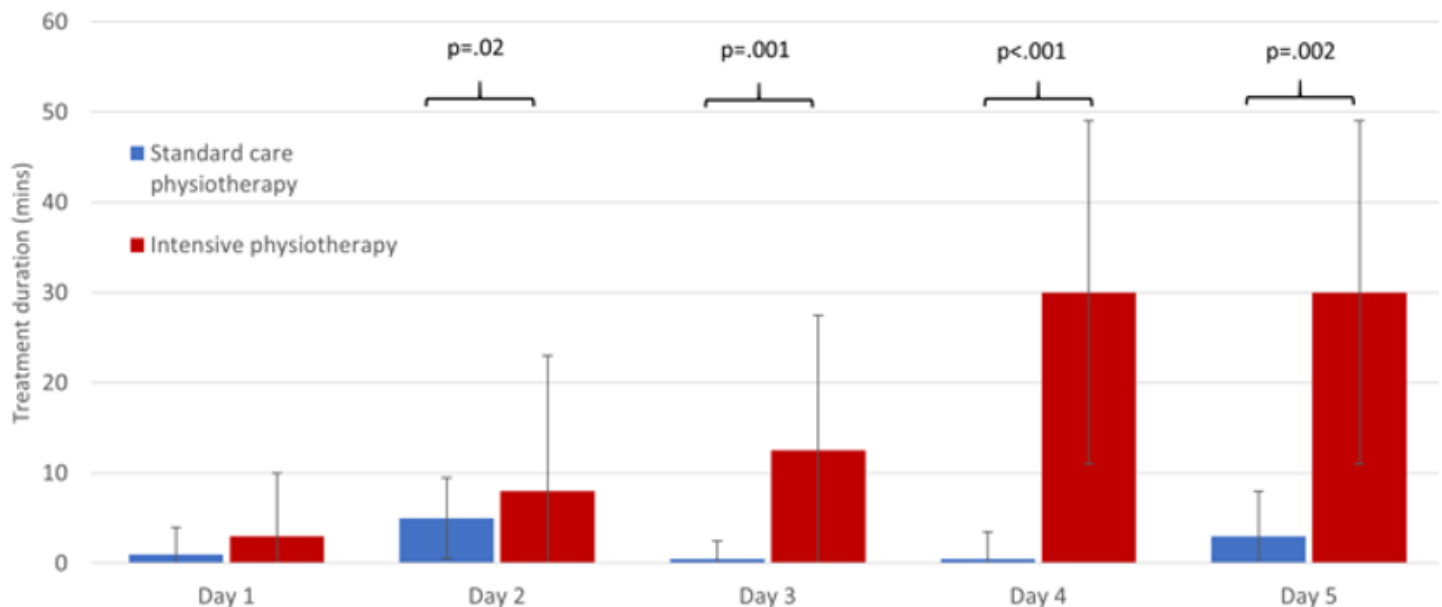

Figure 3: Treatment duration (median +/- IQR) for standard-care physiotherapy vs intensive physiotherapy group patients on days 1 to 5 after emergency abdominal surgery.

\section{Figure 3}

See image above for figure legend

\section{Supplementary Files}

This is a list of supplementary files associated with this preprint. Click to download.

- SupplementalfileTable1.docx 